\title{
Conhecimento dos médicos pediatras e odontopediatras de Bauru e Marília a respeito de flúor
}

\author{
Knowledge of pediatric doctors and dentists \\ from Bauru and Marília about fluoride
}

\author{
Marília Afonso Rabelo Buzalaf 1 \\ Irene Ramires 1 \\ Andréa Gutierrez Maria 2 \\ José Roberto Berber Peres 3 \\ José Roberto Pereira Lauris 4
}

1 Faculdade de Odontologia de Bauru, USP.

Al. Dr. Octávio Pinheiro Brisolla 9-75, Vila Nova,

Cidade Universitária, 17012-901, Bauru SP. mbuzalaf@fob.usp.br

2 Faculdade de Química de Bauru, Unesp.

3 Médico pediatra.

4 Departamento de

Odontopediatria,

Ortodontia e Saúde Coletiva,

Faculdade de Odontologia

de Bauru, USP.
Abstract The aim of this study was to evaluate the knowledge of pediatric doctors and dentists, in respect to the use of fluoridated compounds. Ninety-one pediatric doctors and seventy-two pediatric dentists from Bauru and Marília municipalities were visited. After agreeing in participating, they received a questionnaire with 22 questions. They immediately filled and returned the questionnaires. Data obtained were analyzed by descriptive statistics, using absolute and relative frequencies, represented by tables. Some pediatric drugs containing a combination of vitamins and fluoride are usually prescribed by pediatric doctors, while fluoride gels, varnishes and rinsing solutions are often recommended by pediatric dentists. It was not established a relationship between the knowledge of pediatric doctors and dentists regarding the sources of fluoride intake and gender, time of graduation, age, place of graduation, city and area of working (public, private area or both). The results suggest that the knowledge of pediatric doctors and pediatric dentists that work in Bauru and Marilia, regarding the presence of fluoride in various sources of intake is insufficient and, in some cases, concerning, with respect to the prevention of dental fluorosis.

Key words Pediatric doctors, Dentists, Fluoride, Fluorosis
Resumo A proposta deste trabalho foi verificar o conhecimento dos médicos pediatras e odontopediatras, a respeito dos compostos fluoretados. Para tanto, foram visitados 91 médicos pediatras e 72 odontopediatras dos municípios de Bauru e Marília, que ao concordarem em participar da pesquisa receberam um questionário com 22 questões. O preenchimento e a devolução foram imediatos. Os dados obtidos foram analisados por meio de estatística descritiva, utilizando freqüências absolutas e relativas, representadas através de tabelas. Mediante a análise dos questionários, verificou-se que alguns polivitamínicos que contêm flúor são usualmente prescritos pelos médicos pediatras, ao passo que os géis, vernizes e soluções para bochecho são bastante empregados pelos odontopediatras. Não foi estabelecida relação entre o conhecimento e tempo de formado, a idade do profissional, a universidade de origem, a cidade em que trabalha e a área de atuação (particular, rede pública ou em ambos) desses profissionais. Os resultados obtidos sugerem que o conhecimento dos médicos pediatras e odontopediatras, das cidades de Bauru e de Marília, a respeito da presença de flúor em várias fontes de ingestão se mostrou insuficiente e, em algumas situações, preocupante, com relação à prevenção de fluorose dentária.

Palavras-chave Médicos pediatras, Odontopediatras, Flúor, Fluorose 


\section{Introdução}

Os resultados positivos obtidos mediante o uso de flúor no controle da cárie dentária são indiscutíveis e reconhecidos cientificamente. No entanto, a ingestão excessiva desse composto no período de formação dos dentes pode levar à fluorose dentária, cuja prevalência vem aumentando a partir da década de 1980. Embora haja consenso da relação existente entre o uso do flúor e a redução de cárie dentária, portanto o reconhecimento da sua ação terapêutica, é preciso entender que o flúor é uma substância tóxica e, como tal, pode causar efeitos colaterais quando altas doses são ingeridas agudamente ou, mesmo, baixas doses cronicamente. O efeito tóxico mais comum do uso crônico do flúor é a fluorose dentária (Thylstrup \& Fejerskov $^{1}$, Burt 2 , Whitford ${ }^{3}$, Assis et al. 4 , Buzalaf et al.5).

A fluorose dentária é um distúrbio no processo de formação do dente e caracteriza-se por uma alteração na estrutura do esmalte, provocada pela intoxicação sistêmica de íons flúor, durante a sua formação (Thylstrup \& Fejerskov $^{1}$, Burt ${ }^{2}$, Assis et al.4, Buzalaf et al.5). Sua ocorrência e gravidade podem variar entre os diferentes indivíduos e populações, devido à influência de fatores ambientais e fisiológicos, bem como à maior exposição a diferentes fontes de ingestão de flúor. Entretanto, há uma relação direta entre o aumento da quantidade de flúor ingerido por um indivíduo e a subseqüente incidência e gravidade da fluorose (Burt ${ }^{2}$, Assis et al.4, Buzalaf et al. ${ }^{5}$, Pendrys et al.6, Fejerskov et al.7, Axelsson ${ }^{8}$ ). Tendo um caráter dose-dependente, o aspecto clínico da fluorose está diretamente relacionado à interação das concentrações de flúor no plasma e o tempo (idade da criança e duração da exposição), podendo variar desde linhas brancas finas até um esmalte opaco e de aspecto calcário, que pode se fraturar após a erupção e/ou se pigmentar. Uma vez que a fluorose ocorre durante a amelogênese, a idade de risco para a dentição permanente é de 11 meses a 7 anos de idade (Thylstrup \& Fejerskov1, Burt², Buzalaf et al. ${ }^{5}$, Axelsson 8 , Evans \& Darvell9). A quantidade de ingestão diária de flúor normalmente aceita como ideal para a prevenção da cárie, e segura para a de fluorose, é de 0,05 a 0,07 mg de $\mathrm{F} / \mathrm{Kg}$ massa corporal (Burt ${ }^{2}$, Buzalaf et al. ${ }^{5}$, Levy et al.10).

A partir do século 20, surgiram muitas fontes de ingestão de flúor e, com isso, as crianças passaram a ter maiores possibilidades de exposição ao flúor. Os alimentos, as bebidas, a água de consumo e os produtos odontológicos fluoretados são, atualmente, considerados as maiores fontes de ingestão de flúor para indivíduos acima de 1 ano de idade (Burt ${ }^{2}$, Pendrys et al. 6 , Levy et al.10, Mascarenhas ${ }^{11}$, Buzalaf et al.12, Lima \& Cury13, Buzalaf et al.15, Buzalaf at al.16). É importante salientar que houve uma mudança considerável dos hábitos alimentares das crianças, tendo aumentado o consumo de alimentos e bebidas processados industrialmente (Buzalaf et al.12, Lima \& Cury13, Buzalaf et al.14, Buzalaf et al.15, Buzalaf at al.16, Heller et al.17). Em 1997, foi relatado que $56 \%$ da população dos EUA consome água fluoretada, além de ser difundido o uso de comprimidos, géis, soluções para bochechos e dentifrícios fluoretados, e o consumo de alimentos e bebidas produzidos com águas fluoretada (Heller et al.17). Portanto, ao se considerar alimentos industrializados, a água fluoretada tem um impacto maior na prevalência de fluorose, indiretamente, quando é usada no processamento de leites e outros alimentos, além das bebidas (Burt ${ }^{2}$, Pendrys et al.6, Buzalaf et al.12, Lima \& Cury13, Buzalaf et al.15, Buzalaf et al.16, Heller et al.17, Pang et al.18).

Os profissionais de saúde que trabalham com crianças podem ou não prescrever produtos fluoretados, como suplementos vitamínicos, dentifrícios fluoretados, produtos odontológicos, que por sua vez podem estar se somando as outras fontes de flúor ingeridas pela criança, e assim, contribuir para a ocorrência de fluorose. Considerando a importância desses profissionais para o controle e a orientação da população quanto ao uso adequado das diversas fontes de ingestão de flúor, o objetivo deste trabalho foi avaliar o conhecimento dos mesmos a respeito da correta indicação e utilização dos compostos fluoretados, das suas concentrações apropriadas e do potencial de toxicidade agudo e crônico do flúor.

\section{Metodologia}

Bauru e Marília situam-se na região oeste do estado de São Paulo, sendo que a distância entre elas é de aproximadamente $100 \mathrm{~km}$. Bauru tem uma população de cerca de 316.064 habitantes, que conta com os serviços de 55 médicos pediatras e 48 odontopediatras, além de três faculdades de odontologia. Marília tem 
uma população de 197.342 habitantes, que é atendida por 36 médicos pediatras e 24 odontopediatras. $\mathrm{Na}$ cidade, a população pode contar, também, com os serviços de uma faculdade de odontologia e duas de medicina.

Estabeleceu-se que a amostra deveria envolver todos os médicos pediatras e odontopediatras das cidades de Bauru e de Marília, atuantes na rede pública, particular ou em ambas. Para a obtenção dos nomes e endereços dos médicos pediatras de Bauru e de Marília, foi contatado o Conselho Regional de Medicina do Estado de São Paulo (Cremesp), solicitando-se uma listagem dos profissionais cadastrados. O mesmo procedimento foi adotado em relação ao Conselho Regional de Odontologia do Estado de São Paulo (Crosp) para a obtenção dos dados dos odontopediatras. Segundo o plano diretor, as cidades são divididas em regiões, que foram utilizadas como referência pela pesquisadora para a localização dos profissionais nas diferentes áreas das cidades e para a definição de um roteiro a ser percorrido durante a pesquisa

Foram adotados como critérios de exclusão o profissional ser professor universitário, estar aposentado ou não estar atuando na profissão. As cidades foram incluídas no estudo por conveniência, para facilitar o trabalho da pesquisadora, e também por serem centros formadores de profissionais de saúde.

Para analisar o conhecimento dos médicos pediatras e odontopediatras, de Bauru e de Marília, a respeito de flúor, foram visitados 91 médicos pediatras e 72 odontopediatras. Inicialmente, o profissional recebeu a carta de informações, através da qual era informado e esclarecido a respeito dos objetivos da pesquisa, bem como dos responsáveis pela mesma. Havendo interesse por parte do profissional em se tornar sujeito da pesquisa, o termo de consentimento livre e esclarecido e o questionário estruturado com 22 questões eram entregues para seu preenchimento. Os questionários foram estruturados da seguinte forma: 6 perguntas específicas e 16 comuns a médicos e odontopediatras. As perguntas específicas feitas aos médicos se referem às informações sobre saúde bucal recebidas na formação e à prescrição de polivitamínicos e sua concentração de flúor. Especificamente aos odontopediatras, foi perguntado a respeito do uso de produtos fluoretados (soluções para bochecho, verniz e gel) e suas concentrações de flúor. Como perguntas comuns: gênero; idade; tempo de formado; universidade em que se formaram; área de atuação do profissional (particular, rede pública ou em ambas); se a água de abastecimento público da cidade onde trabalha é fluoretada; qual a concentração de flúor desta água; se a água engarrafada pode conter flúor; a partir de que idade orienta a escovação com dentifrício fluoretado; qual a concentração de flúor dos dentifrícios; se há orientação em relação à quantidade de dentifrício que deve ser usada; se indica o consumo de Toddynho ${ }^{\circledR}$, Neston ${ }^{\circledR}$, Mucilon ${ }^{\circledR}$, Bolachas Danyt's ${ }^{\circledR}$; se tem conhecimento da concentração de flúor desses alimentos; se tem conhecimento a respeito da toxicidade do flúor e das causas da fluorose.

Algumas vezes, o primeiro contato era estabelecido diretamente com os profissionais, mas, em geral, a recepcionista mediava o recebimento e a devolução dos questionários entre o sujeito da pesquisa e a pesquisadora. O questionário era entregue ao sujeito da pesquisa e recolhido logo após o término do seu preenchimento. Antes da devolução, o impresso era colocado em um envelope. Portanto, a forma de coleta de dados não foi feita através de entrevista e nem por via postal.

O preenchimento era realizado sem nenhum tipo de esclarecimento da pesquisadora a respeito das interpretações das perguntas ou da formulação das respostas. Foi estabelecido também que, para evitar constrangimentos, a pesquisadora não deveria ficar por perto durante a leitura e preenchimento do questionário, além de evitar que, de alguma forma, a sua presença pudesse influenciar nas respostas ou, até mesmo, que a pesquisadora e o sujeito da pesquisa acabassem conversando e discutindo o assunto investigado.

A análise das informações obtidas foi feita por meio de estatística descritiva, utilizando-se de freqüências absolutas e relativas, representadas por meio de tabelas.

\section{Resultados}

O total de médicos pediatras e odontopediatras visitados em Bauru foi de 55 e 48 respectivamente, sendo que, destes, 30 médicos e 32 odontopediatras concordaram em participar da pesquisa. Cerca de $45 \%$ dos médicos pediatras e $8,5 \%$ dos odontopediatras de Bauru não quiseram participar da pesquisa.

$\mathrm{Na}$ cidade de Marília, foi estabelecido contato com 36 médicos pediatras e 24 odontope- 
diatras. Destes profissionais, 18 médicos e 12 odontopediatras concordaram em participar da pesquisa, sendo que cerca de $39 \%$ dos médicos pediatras e de $16,5 \%$ dos odontopediatras não concordaram.

O restante dos profissionais foi excluído da amostra seguindo os critérios previamente estabelecidos ou porque não foi encontrado.

Constatou-se que alguns polivitamínicos que contêm flúor são usualmente prescritos pelos médicos pediatras, embora os mesmos tenham afirmado que não prescrevem polivitamínicos fluoretados (Tabela 1).

Os géis, vernizes e soluções para bochecho são bastante empregados pelos odontopediatras de Bauru e de Marília (Tabela 2).

Os resultados obtidos indicam que o conhecimento dos médicos pediatras e odontopediatras a respeito de fluorose, e de outras fontes de ingestão de flúor, é discutível (Tabela 3).

Não foi estabelecida relação entre o conhecimento dos médicos pediatras e odontopediatras a respeito das fontes de ingestão de flúor e o gênero, tempo de formado, a idade, universidade de origem, cidade em que trabalha e área de atuação (particular, rede pública ou em ambas) destes profissionais.

\section{Tabela 1}

Porcentagem de médicos pediatras de Bauru e Marília que prescrevem alguns medicamentos que contêm flúor, em 2004.

\begin{tabular}{lc}
\hline $\begin{array}{l}\text { Medicamento que } \\
\text { contém flúor }\end{array}$ & \% MPED \\
\hline Kalyamon $^{\circledR}$ & 46,8 \\
Calcigenol $^{\circledR}$ & 12,8 \\
Epelyn $^{\circledR}$ & 4,0 \\
Polivitamínicos & 0,0 \\
\hline
\end{tabular}

${ }^{\star} \mathrm{MPED}-$ médicos pediatras

\begin{tabular}{|c|c|}
\hline \multicolumn{2}{|c|}{$\begin{array}{l}\text { Tabela } 2 \\
\text { Porcentagem dos odontopediatras de Bauru } \\
\text { e de Marília que usam alguns produtos } \\
\text { odontológicos fluoretados, em } 2004 \text {. }\end{array}$} \\
\hline Produtos fluoretados & $\%$ OPED $^{\star}$ \\
\hline Gel & 86,3 \\
\hline Verniz & 72,7 \\
\hline Solução / bochecho & 72,7 \\
\hline
\end{tabular}

*OPED - odontopediatras

\section{Discussão}

Uma vez que a fluorose é um distúrbio na formação do dente, em decorrência da ingestão excessiva de flúor durante o seu período de desenvolvimento, a prevenção deve se concentrar em crianças com menos de 6 anos de idade (Thylstrup \& Fejerskov1, Burt ${ }^{2}$, Assis et al.4, Buzalaf et al.5). Evans \& Darvell ${ }^{9}$ sugeriram que o período de risco para a fluorose dos incisivos centrais superiores está compreendido entre 15 e 24 meses, para os meninos, e entre 21 e 30 meses, para as meninas. Recentemente, questionou-se este período para os incisivos centrais superiores (Bardsen ${ }^{19}$ ). Dados recentes mostram a fluorose dentária com ingestão de flúor de menos de $0,04 \mathrm{mg} / \mathrm{kg}$ peso corporal/dia (Fejerskov et al.7). Entretanto, o nível “ótimo" de ingestão sistêmica de flúor, adequado para o controle da cárie dentária e sem o risco de desencadear lesões de fluorose, clinicamente inaceitáveis, ainda não é precisamente conhecido. Ainda assim, quando se pretende determinar este nível, é importante que todas as fontes de ingestão de flúor, sem exceção, sejam consideradas (Burt², Buzalaf et al.12, Buzalaf et al.14, Buzalaf et al.15, Buzalaf et al.16, Ripa ${ }^{20}$ ).

O flúor está presente em muitos medicamentos pediátricos, sem que haja uma finalidade clara. Através da análise da concentração de flúor em 114 medicamentos pediátricos líquidos, verificou-se que $99,12 \%$ dos medicamentos apresentaram flúor total na sua composição, com variações entre 0,0 e 97,8 ppm F. Destes medicamentos, 79,8\% apresentaram uma concentração de $\mathrm{F}<0,1$ ppm; $12,3 \%$ de 0,1-1 ppm F; 3,5\% de 1-10 ppm F; e 4,4\% > 10 ppm F. Após se considerar a dosagem consumida diariamente, $3,5 \%$ dos medicamentos (entre eles vários polivitamínicos) apresentaram flúor total numa concentração igual ou superior à faixa de segurança para uma criança de $12 \mathrm{Kg}$ (Anzai21). No presente trabalho, constatou-se que embora $100 \%$ dos médicos pediatras tenham declarado que não prescrevem polivitamínicos contendo flúor, $46,8 \%$ assinalaram como polivitamínico de eleição o Kalyamon ${ }^{\circledR}$, e $12,8 \%$ associam Kalyamon ${ }^{\circledR}$ ao Calcigenol ${ }^{\circledR}$, sendo que ambos têm altos teores de flúor (Anzai21). Desses profissionais, 25\% informaram a idade em que fazem esta prescrição: de 21 dias a 1 mês de idade, $4,2 \%$; de 3 a 6 meses de idade, $8,3 \%$; de 1 a 3 anos, $12,5 \%$. Os resultados indicam uma provável falta de conhecimento desses profissionais da presença de 
Tabela 3

Conhecimento dos médicos pediatras e odontopediatras, de Bauru e Marília, a respeito de algumas fontes de ingestão e toxicidade do flúor, 2004.

\begin{tabular}{lrr}
\hline Perguntas & \% MPED* & \% OPED $^{* *}$ \\
\hline A água de abastecimento público da sua cidade é fluoretada? & 100 & 100 \\
A água engarrafada pode conter flúor? & 60 & 90 \\
Indica escovação com dentifrício fluoretado? & 96 & 100 \\
Orienta a quantidade de dentifrício a ser colocada na escova? & 46 & 100 \\
Sabe a concentração de flúor dos dentifrícios? & 0 & 60 \\
Recomenda o consumo de Toddynho ${ }^{\circledR}$ ? & 0 & 0 \\
Recomenda o consumo de Neston ${ }^{\circledR}$ ? & 2 & 0 \\
Recomenda o consumo de Mucilon ${ }^{\circledR}$ ? & 32 & 0 \\
Recomenda o consumo de Neston ${ }^{\circledR}$ associado ao Mucilon ${ }^{\circledR} ?$ & 11 & 0 \\
Recomenda o consumo de bolachas Danyt's ${ }^{\circledR}$ ? & 0 & 0 \\
Sabe a concentração de flúor desses alimentos? & 0 & 0 \\
O flúor é um elemento tóxico? & 100 & 98 \\
Conhece as causas da fluorose? & 87 & 100
\end{tabular}

* \% MPED - porcentagem de médicos que responderam sim.

** \% OPED - porcentagem de odontopediatras que responderam sim.

flúor na composição desses medicamentos. Neste aspecto, existe um agravante a ser considerado: esses profissionais somam à prescrição desses medicamentos, a orientação de que alimentos e bebidas sejam preparados com água engarrafada, que por sua vez pode conter flúor, além de recomendarem o consumo de alguns cereais. Portanto, a falta de conhecimento está levando o profissional a indicar várias fontes de ingestão de flúor, predispondo a criança à ocorrência de fluorose dentária.

A maioria dos alimentos tem concentração de flúor menor que 0,5 ppm, com exceção dos produtos à base de frutos do mar e frango, que podem conter altos níveis de flúor, sendo que a inclusão de ossos, pele e conchas nesses produtos, durante o processo de industrialização, contribui para esses valores elevados. Os produtos à base de frango podem conter de $0,6 \mathrm{a}$ $10,5 \mathrm{ppm}$ de flúor (Levy et al.10). As concentrações de flúor presentes nas bebidas, nas quais se incluem sucos de frutas, chás, refrigerantes, entre outros, são decorrentes daquelas encontradas na água usada para o seu preparo. Estas concentrações normalmente variam entre $0,1 \mathrm{a}$ 1,4 ppm, exceto para os chás, que podem chegar a 7 ppm de flúor (Buzalaf et al.12, Buzalaf et al.14, Buzalaf et al.15, Buzalaf et al.16, Pang et al.18, Winkle et al.22, Silva \& Reynolds 23 , Heilman et al.24, Jackson et al.25). No entanto, em alguns alimentos industrializados, comumente consumidos por crianças brasileiras, e produzidos em diferentes regiões, com água fluoreta- da ou não, foram constatadas concentrações elevadas de flúor (Buzalaf et al.12, Buzalaf et al.15, Buzalaf et al.16). Foram analisados três lotes dos cereais Mucilon ${ }^{\circledR}$ e Neston ${ }^{\circledR}$ e de um achocolatado líquido, o Toddynho ${ }^{\circledR}$, entre outros alimentos. Mas, especificamente, estes três alimentos apresentaram uma concentração de flúor de 2,43 $\mu \mathrm{g} / \mathrm{g}, 6,16 \mu \mathrm{g} / \mathrm{g}$ e 1,18 ppm, respectivamente. Considerando que a ingestão “ótima de flúor" é em torno de 0,05 a 0,07 $\mathrm{mg} / \mathrm{kg}$ peso corporal/dia, esses alimentos podem ser considerados como importantes fatores de risco para fluorose dentária, especialmente quando associados com outras fontes de ingestão de flúor. Tomando como exemplo, uma criança de 2 anos de idade, pesando aproximadamente $12 \mathrm{~kg}, 36 \%$ do limite máximo de sua ingestão diária recomendada $(0,07 \mathrm{mg} \mathrm{F} / \mathrm{kg}$ peso corporal) seria alcançado com o consumo destes alimentos apenas uma vez ao dia. De acordo com os resultados deste estudo, 32\% dos médicos recomendam o consumo de $\mathrm{Mu}$ cilon ${ }^{\circledR}$ e $11 \%$ recomendam o consumo associado de Mucilon ${ }^{\circledR}$ e Neston ${ }^{\circledR}$, sendo que $100 \%$ desconhecem a concentração de flúor presente nestes alimentos (Buzalaf et al.12).

A fluoretação da água de abastecimento público das cidades de Bauru e Marília tem sido realizada com base na média anual das temperaturas máximas diárias, não sendo considerado nenhum outro fator para o estabelecimento da concentração de flúor indicada para a comunidade. Sendo assim, a concentração de 
flúor na água de abastecimento público considerada ideal para a região é de 0,7 ppm. Os valores empregados para este cálculo foram preconizados por Galagan \& Vermillion, em 1957, quando os dentifrícios e produtos odontológicos contendo flúor não eram usados em grande escala, o que só passou a acontecer no final do século 20 (Buzalaf et al.14, Ramires26). Além destes, um outro aspecto a ser considerado é a mudança dos hábitos alimentares da população, que ocasionou um aumento de fontes de ingestão de flúor (Buzalaf et al.12, Buzalaf et al.14, Buzalaf et al.15, Buzalaf et al.16). Embora $87 \%$ dos médicos pediatras e $100 \%$ dos odontopediatras tenham declarado saber que a água de abastecimento público de suas cidades é artificialmente fluoretada, apenas 4,2\% dos médicos e $56,8 \%$ dos odontopediatras souberam informar a concentração de flúor presente na água. Cerca de $60 \%$ dos médicos pediatras e 90\% dos odontopediatras de Bauru e de Marília declararam ter conhecimento da presença de flúor na água engarrafada. O preparo dos alimentos com água engarrafada é recomendado por $32 \%$ dos médicos. Embora os resultados indiquem que os profissionais não sabem em que concentração o flúor se encontra presente na água de abastecimento público, na água engarrafada, nos alimentos e nos medicamentos, praticamente $100 \%$ dos profissionais (médicos pediatras e odontopediatras) têm conhecimento de que o flúor pode ser um elemento tóxico.

A utilização cada vez maior das águas engarrafadas faz com que estas se tornem importantes fontes de ingestão sistêmica de flúor, podendo desta forma ser consideradas como fator de risco para fluorose, principalmente quando empregadas no preparo de alimentos para crianças (leites e outros). Embora as águas engarrafadas sejam amplamente comercializadas no Brasil, sua concentração de flúor apresenta grandes variações e diferenças significativas em relação às informações no rótulo $(\mathrm{Bu}-$ zalaf et al.16, Bastos et al.27, Ramires et al.28).

Os resultados deste estudo sugerem que o grau de interesse dos médicos pediatras pelo assunto não é grande, uma vez que $45 \%$ não quiseram participar do estudo. Maltz \& Lacerda 29 pesquisaram a conduta e o grau de conhecimento dos médicos pediatras a respeito da prescrição de flúor, de sua presença na água de abastecimento público e de sua ação. Verificouse que esses profissionais têm pouco conhecimento a respeito do assunto.
No exercício da odontologia, existem alguns produtos fluoretados que são amplamente utilizados na prevenção e no controle da cárie em crianças. Alguns produtos odontológicos fluoretados têm concentrações de flúor variando entre 230 ppm (soluções para bochecho) até $23000 \mathrm{ppm}$ (vernizes fluoretados). As soluções fluoretadas para bochecho indicadas para uso semanal contêm NaF 0,2\% (910 ppm F), e aquelas para uso diário são compostas de $\mathrm{NaF}$ a $0,05 \%$ (230 ppm F). A quantidade de solução ingerida após o bochecho é inversamente relacionada à idade e ao hábito de bochechar, e diretamente proporcional à duração e ao volume usado de solução. Estas soluções, normalmente, são indicadas para a faixa etária de 5 a 6 anos, em programas escolares, quando as crianças já têm controle da deglutição. Com relação à ocorrência de fluorose dentária, sua participação parece ser insignificante (Ripa20, Newbrun ${ }^{30}$, Richards \& Banting ${ }^{31}$ ). Cerca de $72,7 \%$ dos odontopediatras de Bauru e Marília indicam bochecho. Embora façam uso dessas soluções, cerca de $65 \%$ dos profissionais não souberam informar as concentrações de flúor e a idade ideal para se proceder ao bochecho.

O gel fluoretado, utilizado para aplicações tópicas, tem uma concentração de $\mathrm{NaF}$ equivalente a 12.300 ppm F (NaF a 1,23\%). A quantidade de flúor ingerida, após a aplicação, oscila entre 10 a 35 mg F, quando não é usado o aparelho de sucção, e de 2 a $7 \mathrm{mg}$, quando o aparelho é empregado. Embora estas quantidades excedam os limites estabelecidos como "seguros", sua aplicação é realizada com intervalos de 3 a 12 meses. Ainda que não esteja estabelecido seu grau de importância como fator de risco para fluorose, sua indicação é restrita às crianças de alto risco de cárie (Ripa ${ }^{20}$, Newbrun ${ }^{30}$ ). Mesmo com uma indicação restrita, os géis fluoretados são amplamente utilizados pelos odontopediatras de Bauru e de Marília, pois $86,3 \%$ declaram usar estes produtos. Este resultado é preocupante, uma vez que desses profissionais, $22,7 \%$ indicam o uso de gel entre 1 e 3 anos idade, e apenas $6,8 \%$ mencionaram avaliar as condições de risco de cárie para fazer ou não a indicação do gel.

Os vernizes fluoretados são produtos de alta concentração de flúor, em torno de 22.600 ppm F. Entretanto, são considerados seguros, devido à sua rápida aderência sobre a superfície dentária, à lenta dissolução na boca, à lenta liberação de flúor e à pequena quantidade necessária para sua aplicação, que deve ser feita 
apenas por profissionais capacitados. Estes têm indicação precisa: indivíduos de médio a alto risco de cárie (Ripa ${ }^{20}$, Newbrun ${ }^{30}$ ). O uso de vernizes fluoretados é bastante difundido entre os odontopediatras de Bauru e de Marília, cerca de $72,7 \%$. No entanto, o conhecimento destes profissionais com relação à concentração de flúor dos vernizes é preocupante, já que apenas $20 \%$ souberam informar corretamente.

Os dentifrícios, que são os produtos odontológicos mais freqüentemente usados, contêm uma concentração de flúor variando entre 500 e 1500 ppm, tanto na forma de fluoreto de sódio quanto na forma de monofluorfostato de sódio. Considerando que a quantidade média colocada nas escovas, por crianças menores de 6 anos de idade, é de 0,55g por escovação ( $\mathrm{Li}$ ma \& Cury13, Richards \& Banting 31 , Pessan et al.32, Rodrigues et al.33, Almeida et al. ${ }^{34}$ ), se o dentifrício contém 1000 ppm F, isto implica em uma exposição de 0,55 mg de flúor por escovação. Em média, $48 \%$ dessa quantidade é ingerida por crianças de 2 a 3 anos de idade, $42 \%$ por crianças de 4 anos e $34 \%$ por crianças de 5 anos (Richards \& Banting31). No Brasil, estudos conduzidos com crianças de 2 e 3 anos de idade, residentes em áreas com água fluoretada, mostraram que as mesmas ingerem em média $0,061 \mathrm{mg} / \mathrm{kg} / \mathrm{dia}$ de flúor (variação de 0,011 a $0,142)$, a partir do dentifrício; e que o dentifrício contribuía com $55 \%$ da quantidade total de flúor ingerida diariamente (Lima \& Cury13, Paiva et al.35). Portanto, fica evidente que a escovação com dentifrício fluoretado, mesmo com os de 500 ppm F, aumenta significativamente a ingestão diária de flúor, em particular entre as crianças de 2 a 3 anos de idade, pois quanto mais nova a criança, maior a ingestão. Sendo assim, o conhecimento dos profissionais de saúde, que trabalham especificamente com crianças, a respeito desses aspectos relacionados à concentração de flúor e à correta utilização dos dentifrícios fluoretados é fundamental na prevenção da fluorose. Nesse sentido, são preocupantes os resultados da pesquisa com os odontopediatras de Bauru e Marília, no que se refere ao conhecimento destes profissionais sobre a concentração de flúor nos dentifrícios e à idade ideal para se iniciar o uso desses produtos. Embora a legislação que estabelece as normas para a fluoretação dos dentifrícios no Brasil esteja em vigor desde 1989, e este seja um dos principais produtos utilizados na prevenção da cárie dentária em larga escala, 24\% dos odontopediatras não souberam informar a concentração de flúor desses produtos. Além desses, $16 \%$ informaram concentrações erradas; ou seja, cerca de $40 \%$ dos odontopediatras não souberam informar a concentração de flúor dos dentifrícios. Ainda pior é o resultado da idade correta para se iniciar a escovação com dentifrícios fluoretados. A escovação para crianças com menos de 3 anos de idade é recomendada por $43 \%$ dos odontopediatras, que são especialistas no atendimento a crianças. Destes, 17\% indicam escovação com dentifrício fluoretado para crianças com 6 meses de idade, $7 \%$ para crianças de 12 meses, $2 \%$ para crianças de 18 meses e $17 \%$ para crianças com 24 meses de idade. Recomendam iniciar a escovação a partir dos 3 anos de idade, 57\% dos profissionais, sendo: $27 \%$ com 3 anos de idade, $5 \%$ com 4 anos, $10 \%$ com 5 anos e $12 \%$ para crianças com 6 anos de idade.

Tanto os médicos pediatras como os odontopediatras deveriam estar mais bem informados e preparados para orientar corretamente a respeito do uso dos compostos fluoretados recomendados na prática clínica, as concentrações apropriadas e seguras e o potencial de toxicidade agudo e crônico; para que, dessa forma, sua utilização resultasse na máxima eficácia clínica, com o mínimo de risco à saúde do paciente. Os resultados deste estudo mostram que há deficiência de formação e falta de conhecimentos desses profissionais nesta área.

\section{Conclusão}

O conhecimento dos médicos pediatras e odontopediatras, das cidades de Bauru e de Marília, a respeito da presença de flúor em várias fontes de ingestão se mostrou insuficiente e, em algumas situações, preocupante, com relação à prevenção de fluorose dentária. 


\section{Colaboradores}

MAR Buzalaf participou como orientadora e coordenadora da pesquisa. I Ramires participou como co-orientadora do projeto, durante a aplicação dos questionários e tabulação dos dados, na redação do relatório para a Fapesp e do manuscrito. AG Maria participou da elaboração do projeto, tabulação dos resultados, relatórios e manuscrito. JRB Peres, médico, auxiliou na elaboração do questionário e do projeto. JRP Lauris orientou a definição da amostra e os métodos para a tabulação dos resultados estatísticos.

\section{Referências}

1. Thylstrup A, Fejerskov O. Clinical appearance of dental fluorosis in permanent teeth in relation to histological changes. Comm Dent O Epidemiol 1978; 6:329-37.

2. Burt BA. The changing patterns of systemic fluoride intake. J Dent Res 1992; (Special issue) 71:1228-37.

3. Whitford GM. Effects of plasma fluoride and dietary calcium concentrations on GI absorption and secretion of fluoride in the rat. Calcif Tissue Int 1994; 54:421-5.

4. Assis GF, Buzalaf MAR, Faria FAC, Granjeiro JM, Torres AS. Mecanismos biológicos e influência de fatores ambientais na fluorose dentária e a participação do flúor na prevenção da cárie. Revisão de literatura. Rev Fac Odontol Bauru 1999; 7(3/4):63-70.

5. Buzalaf MAR, Cury JA, Whitford GM. Fluoride exposures and dental fluorosis: A literature review. Rev Fac Odontol Bauru 2001; 9:1-10.

6. Pendrys DG, Katz RV, Morse DE. Risk factors for enamel fluorosis en a fluoridated population. Am J Epidemiol 1997; 143:808-15.

7. Fejerskov O, Baelum V, Richards A. Dose-response and dental fluorosis. In: Fejerskov O, Ekstand J, Burt BA. Fluoride in dentistry. Copenhagen: Munksgaard; 1996. p. 153-66.

8. Axelsson P. Use of fluorides. In: Axelsson P. Preventive materials, methods, and programs. Chicago: Quintessence Publishing; 2004. p. 263-368.

9. Evans RW, Darvell BW. Refining the estimate of the critical period for susceptibility to enamel fluorosis in human maxillary central incisors. J Pub Health Dent 1995; 55:238-49.

10. Levy SM, Kritsy MC, Warren JJ. Sources of fluoride intake in children. J Pub Health Dent. Winter 1995; 55:39-52.

11. Mascarenhas AK. Risk of factor for dental fluorosis: a review of the recent literature. Pediatr Dent 2000; 22:269-77.

12. Buzalaf MAR, Granjeiro JM, Damante CA, Ornelas F. Fluoride content of infant formulas prepared with deionized, bottled mineral and fluoridated drinking water. ASDC J Dent Child 2001; 68(1):37-41.

13. Lima YBO, Cury JA. Ingestão de flúor por crianças através da água e dentifrício. Rev Saude Publica 2001; 35:576-81.
14. Buzalaf MAR, Granjeiro JM, Damante CA, Ornelas F. Fluctuations in public water fluoride level in Bauru, Brazil. J Pub Health Dent 2002; 62(3):173-6.

15. Buzalaf MAR, Granjeiro JM, Duarte JL, Taga MLL. Fluoride content of infant foods in Brazil and risk of dental fluorosis. ASDC J Dent Children 2002; 69(2): 196-200.

16. Buzalaf MAR, Damante CA, Trevizani LMM, Granjeiro JM. Risk of fluorosis associated to consumption of infant formulas prepared with bottled water. $J$ Dent Child 2004; 71(2):110-3.

17. Heller KE, Ekuland SA, Burt BA. Dental caries and dental fluorosis at varying water fluoride concentrations. J Pub Health Dent 1997; 57:136-43.

18. Pang DTY, Philipps CL, Bawden JW. Fluoride intake from beverage consumption in a sample of North Carolina children. J Dent Res 1992; 71:1382-8.

19. Bardsen A. "Risk periods" associated with the development of dental fluorosis on maxillary permanent central incisors. Acta Odontol Scand 1999; 57:247-56.

20. Ripa LW. A critique of topical fluoride methods (dentifrices, mouthrinses, operator and self-applied gels) in an era of decreased caries and increased fluorosis prevalence. J Pub Health Dent 1991; 51(1):23-41.

21. Anzai A. Concentração de flúor em medicamentos pediátricos e risco de fluorose dentária [dissertação]. Bauru (SP): Universidade de São Paulo; 2003.

22. Winkle SV, Levy SM, Kiritsy MC, Heilman JR, Wefel JS, Marshal T. Water and formula fluoride concentrations: significance for infants fed formula. Pediatr Dent 1995; 17:305-10.

23. Silva M, Reynolds EC 1996. Fluoride content of infant formula in Australia. Aust Dent J 1996; 41:37-42.

24. Heilman JR, Kiritsy MC, Levy SM, Wefel JS. Assessing fluoride levels of carbonated soft drinks. J Am Dent Assoc 1999; 130:1593-9.

25. Jackson RD, Brizendine EJ, Kelly AS, Hinesley R, Stookey GK, Dunipace AJ. The fluoride content of foods and beverages from negligebly and optimaly fluoridated communities. Comm Dent Oral Epidemiol 2002; 30:382-91.

26. Ramires I. Avaliação da concentração de flúor na água de abastecimento público de Bauru, antes e depois dos procedimentos de fluoretação [dissertação]. Bauru (SP): Universidade de São Paulo; 2004. 
27. Bastos JRM, Buzalaf MAR, Levy FM, Ribeiro TTT, Mazzone FHR. Concentração de flúor em água mineral engarrafada e de fontes naturais das cidades de Lindóia, Águas de Lindóia e Serra Negra, Brasil. Rev Fac Odont Univ Passo Fundo 2001; 6:15-9.

28. Ramires I, Cattani L, Grec RHC, Moura PG, Lauris JRP, Buzalaf MAR. Avaliação da concentração de flúor e consumo de água mineral. Rev Saude Publica 2004; 38:459-65.

29. Maltz M, Lacerda P. Conhecimento do pediatra na área da saúde bucal. Rev Assoc Bras Odontol 2001; 9:210-6.

30. Newbrun E. Current regulations and recommendations concerning water fluoridation, fluoride supplements, and topical fluoride agents. J Dent Res 1992; 71:1255-65.

31. Richards A, Banting DW. Fluoride toothpastes. In: O Fejerskov, J Ekstand, BA Burt . Fluoride in dentistry. Copenhagen: Munksgaard; 1996. p. 328-46.
32. Pessan JP, Silva SMB, Buzalaf MAR. Evaluation of the total fluoride intake of 4-7-year-old children from diet and dentifrice. J App O Science 2003; 11(20):150-6.

33. Rodrigues MHC, Bastos JRM, Buzalaf MAR. Fingernails and toenails as biomarkers of subchronic exposure to fluoride from dentifrice in 2-3-year-old Children. Caries Res 2004; 38:109-14.

34. Almeida BS, Cardoso VES, Buzalaf MA. Fluoride ingestion from toothpaste and diet in 1-3-year-old Brazilian children. Comm Dent O Epidemiol 2006. (In press).

35. Paiva SM, Lima YB, Cury JA. Fluoride intake by Brazilian children from two communities with fluoridated water. Comm Dent O Epidemiol 2003; 31(3): 184-91.

Artigo apresentado em 11/04/2005

Aprovado em 20/10/05

Versão final apresentada em 17/11/05 